\title{
El Deseo de Ser Primera Generación Universitaria. Ingreso y Graduación en Jóvenes de Sectores Populares
}

\author{
The Desire to Be the First University Generation. Admission \\ and Graduation in Young People from Popular Sectors
}

\author{
Joaquín Linne * \\ Universidad de Buenos Aires, Argentina
}

\begin{abstract}
Este artículo indaga las estrategias y dificultades que enfrentan los estudiantes universitarios de sectores populares del Área Metropolitana de Buenos Aires (AMBA). A nivel metodológico, se realizaron observaciones y veinte entrevistas en profundidad a jóvenes de cuatro universidades públicas del AMBA. Entre los resultados, se observa que las razones de deserción se encuentran interrelacionadas: la falta de un espacio propio para estudiar, la mayor distancia de viaje, las trayectorias educativas intermitentes y la falta de referentes cercanos que faciliten la transmisión de capital académico. Por último, se describen los factores que contribuyen al proceso de afiliación institucional, entre los que se destacan el mayor involucramiento institucional y aprovechamiento de los recursos disponibles -bibliotecas y tutorías-, la interacción con el grupo de pares de la institución, el centro de estudiantes y los cursos de ingreso prolongados.
\end{abstract}

Descriptores: Educación; Universidad; Jóvenes; Clase social; Deserción escolar.

This article investigates the strategies and difficulties faced by university students from popular sectors of the Metropolitan Area of Buenos Aires (AMBA). At the methodological level, observations and twenty in-depth interviews were carried out at four public universities of the AMBA. Among the results, it is observed that reasons for desertion are interrelated: the lack of a proper space to study, the greater distance of travel, intermittent educational trajectories and the lack of nearby referents that facilitate the transmission of academic capital. Finally, the factors that contribute to the institutional affiliation process are described, among which stand out the greater institutional involvement and use of available resources -libraries and tutorials-, the interaction with the peer group of the institution, the center of students and extended entrance courses.

Keywords: Education; University; Young; Social class; School dropout.

Los pibes están tratando de encontrar un lugar en un contexto donde hay muchas dificultades. A veces tienen muchas dificultades o trabas para el estudio. Hay muchas pequeñas trabas que terminan perjudicando siempre a los más pobres. (Padre De Vedia, párroco Capilla Caacupé, Villa 21-24, Barracas, Zona Sur CABA)

\section{Introducción}

Este artículo explora dificultades y estrategias de jóvenes del Área Metropolitana de Buenos Aires (AMBA) que son primera generación de universitarios en sus familias. Como argumentan Botinelli y Sleiman (2017), Argentina posee uno de los sistemas de educación

*Contacto: joaquinlinne@gmail.com

ISSN: 0718-7378

www.rinace.net/rlei/
Recibido: 23/11/2017

$1^{\text {a }}$ Evaluación: 15/12/2017

Aceptado: $\quad 14 / 02 / 2018$ 
superior con mayor prestigio mundial, dado que combina un alto nivel académico y de investigación científica, con acceso irrestricto, libre y gratuito. El sistema cuenta con 57 universidades públicas y 66 privadas. De los dos millones de estudiantes universitarios, el $80 \%$ se concentra en las públicas. Si bien en los últimos años se ha incrementado la población que cursa estudios superiores, continúa siendo un desafío elevar su relativamente baja tasa de graduación.

El trabajo que aquí se presenta surgió a partir de mi experiencia docente en el curso de ingreso de la Universidad Nacional de Lanús (UNLA) y en la sede Lugano del Ciclo Básico Común de la Universidad de Buenos Aires (UBA). Algunas preguntas que orientaron la investigación fueron las siguientes. ¿En qué medida las universidades se hallan preparadas para acompañar a los estudiantes de sectores populares? ¿Cuáles son aceptados por las instituciones, y cuáles expulsados? ¿Por qué abandonan los que abandonan? ¿Las universidades justifican con la meritocracia el cierre social y la "selección" de graduados? ¿Cómo se despliega en esos espacios la tensión entre ser reproductora de estructuras sociales, y funcionar como una herramienta de empoderamiento para cientos de miles de jóvenes?

\section{Revisión de la literatura}

\subsection{El origen social como condicionante de las trayectorias educativas}

A partir de investigar el sistema educativo francés, Bourdieu y Passeron (2003) señalan de modo pionero que el nivel socioeconómico opera como condicionante fundamental en las trayectorias universitarias. Las instituciones académicas reproducen de algún modo las estructuras sociales. A través de estrategias de diferenciación y distinción, favorecen a que los graduados con mejores calificaciones sean de sectores medios y altos. En este sentido, quienes habitan en hogares con jefes de familia profesionales viven la institución escolar como una proyección de las prácticas que atravesaban en su esfera doméstica.

Coulon (2005, citado en Wallach y Vidondo, 2017) propone analizar el ingreso universitario como un pasaje con tres períodos: el de extranjeridad, en el que se accede a un ámbito desconocido; el de aprendizaje, que implica el proceso de adaptación e incorporación de normas y códigos básicos de la institución; y el de afiliación, que alude a las prácticas interpretativas, críticas y estratégicas que permiten "apropiarse" de la institución y sentirse parte de ella. Wallach y Vidondo (2017) aclaran que la duración de estos períodos depende de cada experiencia educativa, debido a que se intersectan trayectorias individuales e institucionales. Siguiendo a Coulon (2005), Manuale (2013) sostiene que afiliarse significa convertirse en miembro de la institución universitaria, en un proceso intelectual e institucional que implica asimilar la información implícita y apropiarse de las rutinas ocultas que son necesarias para sobrevivir en la institución.

A nivel regional, estos enfoques son retomados por diversos autores. Sito y Kleiman (2017) investigan las políticas afirmativas en universidades de Brasil y Colombia, entendidas como aquellas que establecen un cupo mínimo de estudiantes indígenas o afroamericanos, que autores europeos o estadounidenses denominan "estudiantes no tradicionales". Este grupo experimenta conflictos en el proceso de apropiación de la lectoescritura académica, dado que deben decodificar mecanismos institucionales implícitos y no dichos. El fenómeno de la deserción se evidencia a nivel global en estudios como el de Rojas (2009), quien menciona que abandonan más de la mitad de los estudiantes 
en universidades de Colombia, o en el caso de Barcelona, donde Sánchez-Gelabert y Andreu (2017) sostienen que quienes más dejan la universidad son estudiantes no tradicionales.

El aumento relativo de las oportunidades para acceder a la educación superior ocurrida en los últimos 50 años ha tenido como resultado una heterogeneidad creciente del perfil de estudiantes. Esto ha repercutido en el acceso a la universidad de ciertos colectivos que históricamente no habian participado en educación superior. La literatura científica les ha identificado como estudiantes no tradicionales, por ser colectivos atípicos o con poca participación en la universidad. (Sánchez-Gelabert y Andreu, 2017, p. 28)

Existe una marcada relación entre las probabilidades de no terminar los estudios y las decisiones de abandono y el perfil de estudiantes no tradicionales (Milesi, 2010). Estas diferencias se fundamentan en que a estudiantes no tradicionales les afectan más las variables no académicas: compromisos laborales y familiares, tiempo de viaje, migraciones y dificultades socioeconómicas. En consecuencia, sus experiencias académicas se caracterizan por tener menos intensidad y duración con los espacios, actores y recursos de socialización institucionales (Kessler, 2002).

Estos hallazgos ponen de manifiesto que la manera de pensar y vivenciar la universidad varía de manera notable según corresponda a estudiantes tradicionales o no tradicionales. Es por esto que, en paralelo al aumento de la tasa de estudiantes universitarios no tradicionales, se incrementó la tasa de deserción, en particular en aquellas instituciones que más población no tradicional poseen (Daza, 2013; Elias, 2008; Laing y Robinson, 2003; Sánchez-Gelabert y Andreu, 2017).

Las trayectorias de los denominados estudiantes no tradicionales son comunes a las aquí descriptas para la población mayoritaria de las universidades del Conurbano bonaerense: estudiantes de nuevo tipo, en ocasiones inmigrantes o migrantes internos, de sectores populares, con trayectorias educativas no lineales y de menor intensidad; a veces de mayor edad, mujeres sostén de familia y primeras generaciones universitarias. En este sentido, la ampliación del acceso a la educación pública no implica la neutralización de los efectos de las desigualdades socioeconómicas y culturales. Ezcurra (2011) acuña para este fenómeno la expresión "procesos de inclusión excluyente” y Saraví (2015) la de "inclusión desigual”, observando en numerosas ocasiones que las instituciones, en vez de neutralizar o disminuir las desigualdades de origen, las refuerzan.

En Argentina, Tiramonti (2004), Chaves, Fuentes y Vecino (2016) y Nobile (2016) coinciden en que los segmentos de escolarización diversos brindan calidades educativas disímiles y, por ende, establecen horizontes de expectativas de acuerdo al nivel socioeconómico estudiantil. Esta segmentación ocasiona desiguales distribuciones de oportunidades. Carabaña (2013), Daza (2013) y Sánchez-Gelabert y Andreu (2017) arriban a conclusiones similares para el caso de España.

Ante la masificación universitaria producida durante las últimas décadas, se han desplegado ciertos mecanismos de "selectividad social" (Arias et al., 2017; Gluz, 2011). Aun con el ingreso irrestricto o semi-irrestricto, las universidades continúan generando cierto cupo o "filtro" (Zandomeni et al., 2016). A nivel formal, cursos eliminatorios, cupos por carrera, exámenes de admisión. A nivel informal, cursos de ingreso supuestamente nivelatorios e inclusivos, como el del CBC de la UBA, se convierten en importantes "filtros", dado que sólo la mitad de los estudiantes que lo cursan lo terminan en un año (Arias et al., 
2017). Esto se justifica en parte por la escasez de recursos y también por el imaginario liberal de que, a mayor inclusión, menor calidad académica (Chiroleu, 2016).

Pierella (2016) postula que el origen social y el colegio de procedencia influyen en los procesos de afiliación a la universidad. Quienes son hijos de universitarios tienden a portar como mandato socio-familiar realizar una carrera universitaria, motivados por ideales proyectados desde su niñez y adolescencia, experimentando líneas de afinidad entre mandato familiar, biografía escolar y proyecto institucionalizado. Según Busso y Pérez (2015), gran parte de los jóvenes posee un proyecto de estudio pos-secundario: desde ir a una universidad o terciario hasta realizar cursos de idiomas, computación o adquisición de oficios. Este mandato por perfeccionarse se encuentra asociado al prestigio social, las trayectorias familiares y las constantes exigencias que impone el mercado laboral para acceder a empleos de calidad.

Más allá de las dificultades institucionales para nivelar los diversos capitales culturales, los estudiantes tienden a autorresponsabilizarse por sus deserciones. Bajo la categoría "abandonos voluntarios" en numerosas ocasiones quedan invisibilizadas las "expulsiones institucionales" (Tinto, 2005). En una línea similar, Habley y McClanahan (2004) para el caso estadounidense exponen que las instituciones suelen atribuir la deserción a causas previas, así como a razones personales, voluntarias o extra institucionales de cada estudiante, y rara vez a sus cursos, currículas, planteles docentes, difusión de información institucional, instalaciones o mecanismos de retención universitaria.

Tiramonti (2004) y García de Fanelli (2014) sostienen que la desigualdad educativa comienza en el ciclo inicial. Esta brecha se profundiza en el nivel medio. Pese a la gratuidad de la educación pública y a la ampliación de las tasas de matriculación universitaria en sectores populares, el mayor porcentaje de graduados continúa siendo de sectores medios y altos (García de Fanelli y Adrogué, 2015). Según datos de 2010, Mayer y Cerezo (2017) señalan que, de cada 100 estudiantes inscriptos a universidades públicas, sólo 24 lograron graduarse. De estos últimos, sólo una minoría proviene de sectores populares, dado que el discurso académico suele resultarles más ajeno. Si bien declaran el deseo de ser la primera generación familiar universitaria, esta carga también imprime más soledad a sus experiencias.

Los códigos, normas de la universidad, que los que pasamos por estudios de nivel superior de alguna manera tenemos naturalizados e incorporados, forman parte de un mundo desconocido y muchas veces intimidante. En efecto, ser el/la primero/a que va a la universidad en la familia, en el grupo de amigos/amigas, en el barrio, significa muchas veces que no se tienen modelos cercanos que hayan podido, aunque sea discursivamente, acercar la institución, contando de qué se trata, transmitiendo sus propias experiencias. Esta transmisión es valiosa porque permite que se vayan armando anticipaciones y escenarios posibles de lo que puede ir sucediendo en el proceso subjetivo que significa consolidarse como estudiante universitario. (Wallach y Vidondo, 2017, p. 3)

Como señala el citado trabajo, el capital social académico suele ser más predominante en sectores medios que en populares. Mientras en sectores medios y altos tienen a quién consultar (padres, hermanos) y una disponibilidad para estudiar más amplia, en sectores populares poseen menos recursos y más exigencias familiares.

A su vez, Olave, Rojas y Cisneros (2013) sostienen que las universidades dan por supuesto cierto nivel general de lectoescritura, y que esto acarrea el abandono de estudiantes de sectores populares. Carlino (2005) subraya que no sólo las instituciones dan por supuesto 
cierto nivel general de lectoescritura, sino que además existen "estilos" de escritura propios de cada disciplina, que son inconscientemente evaluados por los docentes pero que no son enseñados de modo explícito. Como consecuencia de estas diferencias de capitales y "moratorias sociales" (Margulis y Urresti, 1996), quienes aspiran a convertirse en estudiantes universitarios arriban con disímiles formaciones, motivaciones, recorridos, recursos y edades al término del colegio secundario.

\subsection{Las universidades del Conurbano bonaerense y el desafío de la inclusión}

Como señalan Botinelli y Sleiman (2017), hasta principios del siglo XX el sistema universitario argentino estaba conformado sólo por las denominadas macrouniversidades: la Universidad Nacional de Córdoba (1613), la UBA (1821) y la UNLP (1905). Con el fin de descentralizar el sistema, el Estado creó ocho nuevas universidades nacionales entre 1919 y 1968: Litoral (1919), Tucumán (1914), Cuyo (1939), Nordeste (1956), Sur (1956), UTN (1959), Neuquén (que en 1971 se refunda como Comahue) y Rosario (1968).

En la década de 1970, con una población nacional de 25 millones de habitantes y sólo 10 universidades nacionales, el Estado, a través del Plan Taquini, decide crear trece más: en 1971 crea la de Río Cuarto; en 1972, las de Lomas de Zamora, Catamarca y Salta (1972); en 1973 las de Luján, San Juan, San Luis, Santiago del Estero, Entre Ríos, La Pampa, Jujuy, Misiones y Luján; por último, en 1975 funda la de Mar del Plata. Una década y media después comenzó un nuevo ciclo de ampliación del sistema universitario: entre fines de la década de 1980 y mediados de la década de 1990, El Estado crea otras diez universidades nacionales: Formosa (1988), La Matanza (1989), Quilmes (1989), San Martín (1992), General Sarmiento (1993), La Rioja (1993), Patagonia Austral (1994), Villa María (1995), Tres de Febrero (1995) y Lanús (1995).

El tercer ciclo de ampliación del sistema universitario se desarrolló entre 2002 y 2015. Con la inauguración de 17 universidades nacionales, se logró el objetivo de que cada provincia cuente con al menos una universidad pública. Tanto la SPU (2016) como Gorostiaga y otros (2017) y Botinelli y Sleiman (2017) sostienen que la creación de nuevas universidades públicas aumentó la tasa de escolarización superior y contribuyó a descentralizar el sistema universitario, que cuenta con poco más de dos millones de estudiantes, de los cuales $80 \%$ concurre a universidades públicas. De éstos, la matrícula que más se incrementó fue la de universidades del Conurbano (un 39\% entre 2008 y 2015), contando con 41.000 nuevos estudiantes que provienen de sectores populares (Botinelli y Sleiman, 2017).

Esta ampliación del sistema focalizada en el Conurbano contribuyó a aumentar las opciones para jóvenes y adultos del AMBA y elevó las tasas de matrícula universitaria en todos los sectores sociales de la región (Botinelli y Sleiman, 2017; SPU, 2016). Algunas características que aportaron estas instituciones fueron mayores opciones de carreras y un despliegue de ofertas curriculares innovadoras, como títulos intermedios, carreras a distancia y semipresenciales, así como ciclos de complementación curricular a quienes poseen terciarios y aspiran a obtener una licenciatura de grado. Asimismo, articulan distintas actividades de extensión como cursos de cultura, oficios e idiomas para adultos mayores, así como ofrecen guarderías maternales, becas y actividades de difusión en colegios secundarios y ferias estudiantiles (Mónaco y Herrera, 2015).

García de Fanelli y Adrogué (2015) señalan que Argentina cuenta con la tasa de estudiantes de nivel superior más alta de la región: $76 \%$ entre la población de 20 a 24 años. 
Las investigadoras plantean que esto es consecuencia de múltiples factores: gratuidad en estudios de grado, mecanismos de admisión no selectivos y amplia regionalización de la oferta en las últimas dos décadas. Pese a esto, observan que las tasas de graduación continúan siendo desiguales según sector social, dada la brecha de capitales culturales y socioeconómicos entre jóvenes que se inscriben al nivel superior. En esta línea, Otero y Corica (2017) argumentan que la principal ampliación de la matrícula universitaria se ubica en el crecimiento de las tasas de acceso, no tanto en la permanencia y el egreso.

En pos de disminuir la deserción durante los primeros años, las universidades públicas del Conurbano suelen ofrecer un curso de ingreso con al menos una materia orientada a nivelar y reforzar la lectoescritura y la metodología de estudio. Además, estas instituciones suelen contar con un sistema de docentes orientadores y tutores, cuya misión es colaborar con información institucional y formación académica. No obstante, Gorostiaga y otros (2017) advierten que, en numerosos casos, los estudiantes no acceden a estas herramientas, o los cursos de ingreso no alcanzan a nivelar las desiguales trayectorias previas. En la misma línea, Wallach y Vidondo (2017) mencionan que gran parte de los inscriptos e ingresantes de sectores populares asocia la universidad a un mundo ajeno, tanto material como simbólicamente. Estas poblaciones suelen creer que la universidad es para una élite socioeconómica y académica.

En esta línea, Bracchi (2016) sostiene que los estudiantes universitarios "exitosos" son aquellos que alcanzan a descifrar y comprender las culturas académicas, que se presentan opacas para amplias franjas de la población estudiantil, en particular, en sectores populares. En este sentido, quienes provienen de familias vinculadas a la cultura universitaria, y especialmente los estudiantes socializados en colegios preuniversitarios, suelen poseer una ventaja o hándicap de cultura académica, entendido como un mayor background en torno a conocimientos y prácticas "socialmente legítimas" (capital cultural), que pueden utilizar como caja de herramientas al momento de producir tareas y enfrentarse a exámenes universitarios.

\section{Metodología}

El objetivo de este artículo es contribuir a los estudios sobre ingreso, permanencia y graduación universitaria en sectores populares, un área de relativa vacancia en la región. Los objetivos específicos son los siguientes: a) indagar las modalidades de las universidades nacionales del AMBA en cuanto a sus cursos de ingreso; b) rastrear las estrategias que despliegan los estudiantes de sectores populares durante su permanencia en la universidad; c) explorar las dificultades asociadas a la deserción, tanto en el ingreso como en las instancias de grado; y d) indagar las problemáticas en torno a la graduación universitaria en sectores populares.

A nivel metodológico, en esta investigación exploratoria y cualitativa he realizado observaciones participantes en distintas instituciones, tanto en UNLA como en UBA. Además, hice veinte entrevistas en profundidad a estudiantes de universidades públicas del AMBA (10 mujeres, 10 varones). El trabajo de campo, realizado entre 2015 y 2017, se focalizó en cuatro universidades públicas: UBA, Universidad Nacional de Lomas de Zamora (UNLZ), UNLA y Universidad Nacional de Avellaneda (UNDAV). Si bien la muestra es intencional y los datos no son extrapolables al conjunto, considero que el trabajo puede 
resultar útil con el fin de describir tendencias, así como indagar y analizar tensiones y desafíos presentes en las universidades públicas.

Siguiendo indicadores del Instituto Nacional de Estadística y Censo (INDEC) (2012), se define a los jóvenes como quienes tienen entre 18 y 30 años de edad. Se categoriza a quienes pertenecen a sectores populares como aquellos que poseen padres con nivel educativo de colegio secundario completo o menor, y residen en hogares que carecen de al menos un servicio público básico. Los jóvenes de sectores medios son caracterizados por tener padres cuyo nivel de estudios alcanzado es al menos de terciario/universitario incompleto y residen en hogares con todos los servicios públicos básicos: agua potable, gas de red, desagüe cloacal, cercanía de colegios, comisarías y hospitales, pavimentación, agua, barrido y limpieza.

Las entrevistas en profundidad tuvieron una extensión aproximada de 45 minutos. Los sujetos de la muestra intencional, quienes poseen entre 18 y 30 años, accedieron a participar de la investigación de modo voluntario. La guía de preguntas se orientó en función de los objetivos; se comenzó por preguntar sobre nivel socioeconómico y luego se pasó a indagar dificultades y estrategias respecto a ingreso, permanencia y graduación. Los datos cualitativos fueron complementados con fuentes secundarias estadísticas, como por ejemplo las del INDEC y las de la Secretaría de Políticas Universitarias (SPU).

Dada la dificultad de acceso a esta población, la selección de los casos fue intencional y aleatoria; a medida que se avanzaba en el trabajo de campo, se recurrió a la técnica de la "bola de nieve" para ampliar el número de casos. Para el análisis de los datos, se tomó de la teoría fundamentada (Glaser y Strauss, 1967) el criterio de "saturación teórica". Así, quedaron establecidas una serie de categorías analíticas que permitieron avanzar en la construcción de los resultados.

\section{Resultados}

\subsection{Los perfiles de estudiantes de sectores populares}

De acuerdo a la SPU (2016), el 78\% de estudiantes y graduados del AMBA pertenecen a universidades públicas, mientras que sólo el $22 \%$ concurre a privadas. Cuando sólo se tiene en cuenta el número de graduados, la proporción entre instituciones públicas y privadas varía levemente: $75 \%$ se gradúan de públicas y $25 \%$ de privadas. Respecto a la distribución por sexo, el $70 \%$ de la población universitaria de estudiantes son mujeres. En cuanto a graduados, la proporción se divide en 50\% entre varones y mujeres. Este dato mostraría que las mujeres poseen más dificultades para graduarse.

Una significativa parte de jóvenes de sectores populares consultados elige estas universidades por la cercanía, la oferta de carreras novedosas, horarios flexibles, carreras a distancia o semipresenciales, recomendación de amistades y familiares, clases de promoción en colegios secundarios y experiencias fallidas en instituciones públicas tradicionales y en privadas. Algunos estudiantes valoran el trato personalizado, el vínculo afectivo que construyen con ciertos docentes y el sentido de comunidad. En primer lugar, se enfatiza la gratuidad del acceso y la ayuda que proveen los programas de tutorías. En segundo lugar, valorizan las becas para apuntes y viáticos.

Creo que es un acceso muy fácil para que, como yo, que no tengo familia que haya ido a la universidad, tal vez porque no habia tantas universidades públicas y gratuitas. Que los pibes puedan entrar a estudiar y ser los primeros en su familia en tener un 
título universitario es un gran paso. Yo, por ejemplo, voy a ser la primera universitaria en mi familia, es mi deseo desde hace tiempo y ya me falta poco. (María, 28, UNLA)

Me gusta esta universidad porque tiene un trato más cercano entre docentes y estudiantes. Tiene mucha articulación con la comunidad en la que se encuentra inmersa. $Y$ trata de ser inclusiva, atendiendo los problemas de la sociedad y generando carreras de grado que puedan aportar soluciones. La UNLA, donde estudié un tiempo, también comparte estas características. (Luciana, UNDAV, 27)

Carlos (30) comenzó a estudiar Ingeniería en la UTN mientras trabajaba. Por los horarios únicos y extensos de cursada, dice que era "imposible" hacerla trabajando. Tiempo después, comenzó Derecho en la UBA mientras trabajaba en un depósito catorce horas por día. No pudo seguir el ritmo, por lo que volvió a abandonar. En el 2010 se inscribió en la UNLA en la carrera de Trabajo social. Ahora sólo le quedan dos materias para recibirse de licenciado. Será el primer graduado de su familia, comenta con orgullo. Si bien no pudo cumplir con el tiempo “ideal” de cursada de cinco años (que la mayoría no logra cumplir), soportó la doble exigencia de compaginar ritmo y tiempos laborales con académicos.

Luciana (27) cursa Bioquímica en la UNDAV y los padres se esfuerzan para que estudie sin trabajar. Sólo trabaja de moza los fines de semana. Para que pueda seguir estudiando, han sido claves las becas de apuntes y viáticos que ha recibido de la universidad durante gran parte de la cursada. Débora (22) cursa por la mañana para ser contadora en la UBA, desde el mediodía trabaja de secretaria en la empresa del padre en Avellaneda, y después vuelve a cursar a la noche. Walter (26) trabaja de cadete y es estudiante avanzado de Diseño de marcas y envasas en la UNDAV. Julián (25) cursa Comercio internacional y aduanas en la UNLZ y trabaja de telemarketer. Andrea (27) fue la única de su división del colegio que siguió estudiando en la universidad. Primero estudió Enfermería en un instituto terciario. Ahora, mientras trabaja de enfermera por las noches, cursa el último tramo de Licenciatura en Enfermería en la UNLA. Mariano (26) estudia diseño industrial en la UNDAV y hace changas cuando puede. Micaela (23) cursa Relaciones laborales en la UNLZ y trabaja de secretaría. Jorge (25) trabaja en la cocina de un restaurante y cursa Licenciatura en alimentos, también en la UNLA. Germán (24) tiene aprobadas la mitad de las materias de Licenciatura en gestión de la calidad de alimentos en la UNLZ y por las noches trabaja en una pizzería. Romina (28) estudia trabajo social en la UBA y trabaja en una empresa de logística. Lorena (26) cursa Turismo en la UNDAV y trabaja de promotora.

Un sujeto específico de la muestra son las mujeres que, luego de haber sido madres, retoman sus estudios. Como indica el anuario estadístico del SPU (2016), las mujeres presentan mayores dificultades que los varones para graduarse. Esto se debe en gran parte a que, como explican los estudios de género, suelen cargar con más responsabilidades familiares y domésticas. Julia (30) es madre de dos hijos y se encuentra en el primer año de abogacía de la UBA. "Ahora que están grandes puedo volver a estudiar", expresa. A María (28) le quedan cinco materias para recibirse de Licenciada en Diseño Industrial en la UNLA. Tiene una hija de cuatro años, está separada, vive con la madre y trabaja de operaria textil en la pequeña empresa que creó un compañero de la universidad. Recibe becas de ayuda económica de la universidad y, a cambio, colabora como tutora par de estudiantes de primer año.

Numerosos casos similares a los descriptos han sido relevados durante esta investigación exploratoria. Si bien una gran parte de estudiantes que son primera generación universitaria enfrentan múltiples dificultades para cursar, también experimentan la 
presión social y familiar de tener que seguir estudiando para "tener un futuro". En este sentido, Germán (24, UNLZ) reflexiona que "hoy hasta para ser cartonero necesitas al menos tener la secundaria completa”.

Como se observa en el trabajo de campo y en la revisión del estado del arte, existen ciertas características comunes entre los estudiantes de universidades del Conurbano: una significativa parte son mayores de 25 años (SPU, 2016), trabaja un promedio de ocho horas por día, realizó trayectorias escolares de "baja intensidad" (Kessler, 2002), hace varios años dejó de estudiar, han formado su propia familia y poseen experiencias de nivel terciario completas o universitarias incompletas. Esto implica que no suelen tener tan incorporados ciertos hábitos de estudio y lectoescritura académica. Estos son dos de los principales motivos que, como se muestra en el siguiente apartado, dificultan su ingreso y afiliación a la universidad.

\subsection{Razones extra-académicas y académicas de deserción}

Una gran parte de los estudiantes entrevistados teme encontrar dificultades durante su experiencia universitaria. También confían en poder sobreponerse a los obstáculos y hallar su vocación en la universidad, graduarse y trabajar de lo que eligieron estudiar. Si los fenómenos sociales tienden a ser multicausales, también lo es la deserción universitaria. En este sentido, entre las múltiples causas de abandono, del trabajo de campo se desprenden tanto razones extra-académicas como académicas, que se encuentran entrelazadas.

A nivel extra-académico, más allá de las diferentes trayectorias y recursos con los que cuenta cada estudiante, el gran condicionante es el socioeconómico. Diversos entrevistados sostienen que no podrían pagar universidades privadas y que, aun contando con la gratuidad de las instituciones públicas, a veces les resulta difícil mantener la economía familiar-doméstica, y además disponer de tiempo y recursos para concurrir a la universidad. Respecto al abandono universitario, comentan que gran parte de sus ex compañeros de colegio no estudian ni trabajan, que al menos deberían hacer cursos de oficio, aprovechar el tiempo, y que otros fueron padres muy jóvenes y tuvieron que salir a trabajar y dejar los estudios.

\section{La educación es un derecho y debería ser una obligación también. Conozco compañeros que terminaron la secundaria y hoy en día están a la deriva. Más que nada cuando no se trabaja, creo que habría que hacer un curso obligatorio de oficios. (Walter, 26, UNDAV) \\ La mayoría de mis compañeros de colegio no siguieron estudiando. Empezaron a trabajar o tuvieron hijos y ya no les interesó hacer una carrera. Algunos se arrepienten de no haber seguido estudiando, pero dicen que ahora les cuesta más retomar, entre la familia, el trabajo y la economía que no ayuda. (Carlos, 30, UNLA)}

Otra razón extra-académica se vincula a las dificultades para combinar tareas académicas con laborales y familiares. La triple exigencia que implica tener una familia, un horario laboral extenso y un prolongado tiempo de viaje suele atentar contra sus proyectos universitarios. En el caso de las mujeres, se suma que la mayor parte de las responsabilidades familiares recaen sobre ellas.

La distancia respecto a la universidad se vislumbra como uno de los principales motivos de deserción. Más allá del sector social, para quienes residen en el Conurbano estudiar en una universidad de la CABA suele demandarles hasta seis horas de viaje diario en transporte público. De hecho, numerosos estudiantes enfatizan haber elegido una 
universidad bonaerense porque les queda a menos de media hora de viaje, mientras que estudiar en la UBA les requería entre dos y seis horas.

También resulta central la carencia de un espacio propio para el estudio. Al vivir con frecuencia en hogares con menor cantidad de ambientes per cápita, por el alto costo de los inmuebles y del metro cuadrado en el AMBA (INDEC, 2012), en sectores populares suelen carecer de un espacio o habitación propia. Este fenómeno de que el tiempo de estudio tienda a limitarse a lo que ocurre en la institución educativa comienza en la educación inicial y se profundiza a partir del nivel medio, tanto a nivel nacional como latinoamericano (Núñez y Litichever, 2016; Saraví, 2015). Tal carencia tiene como consecuencia una recurrente dificultad para concentrarse en leer y resumir apuntes, realizar trabajos domiciliarios y desarrollar un método de estudio propio.

\begin{abstract}
Me cuesta concentrarme en leer en casa porque no tengo un espacio propio. $\Upsilon$ mi hermano, que es más chico, está todo el día jugando videojuegos en el cuarto. Así que trato de estudiar en la biblioteca cuando puedo o de juntarme en lo de algún compañero que tenga más lugar. (Jorge, 25, UNLA)

En mi casa no tengo un lugar tranquilo para estudiar así que vengo a la universidad o voy a bibliotecas o nos juntamos con compañeros en bares, o a veces en el bar de la facultad o en algún aula vacía o en otro espacio que se pueda. (Andrea, 27, UNLA)
\end{abstract}

Otra razón extra-académica es el uso más limitado de las tecnologías de información y comunicación (TIC). Los estudiantes de sectores populares tienden a disponer de menor cantidad y calidad de dispositivos digitales, al mismo tiempo que poseen menos referentes para realizar consultas sobre usos académicos de las TIC. En particular, presentan limitaciones para buscar fuentes de información diversas, manejar procesadores de textos y otros programas vinculados a tareas escolares (Urresti, Linne y Basile, 2015).

A nivel académico, las trayectorias educativas intermitentes o de "baja intensidad" suelen ser un factor que eleva la dificultad para avanzar en el sistema. Gluz (2011) y Grandoli (2011) reconocen que la repitencia y la sobreedad en el nivel medio aumentan en gran medida las posibilidades de deserción universitaria. En efecto, quienes han repetido al menos un año durante el nivel medio tienden a abandonar la universidad con mayor frecuencia que quienes han realizado trayectorias continuas.

En el trabajo de campo he observado que la deserción y la sobreedad aumentan a medida que disminuye el nivel socioeconómico, y las probabilidades de promocionar materias se elevan a medida que aumenta el nivel socioeconómico. No obstante, es notable el número de ingresantes a las universidades del Conurbano que ya poseen una vida laboral, a veces profesional, y que buscan un título para consolidar esa trayectoria. De éstos, una gran parte no fue a la UBA porque les queda lejos, la presumen como demasiado elitista y exigente, o porque la oferta horaria no es amigable con alguien que trabaja y vive en el Conurbano.

En mi casa es muy complicado el tema de la economía, entonces cada tanto ando cargando la Sube [tarjeta del transporte público] o ando con el saldo negativo y acogotada. Pero creo que capaz que eso en otra universidad tendría que poner para la universidad y no tendría para el pasaje, o no tendría para pagar la cuota y sí para el pasaje. Eso es lo bueno de que sea público y gratuito. (Julián, 25, UNLZ)

Las trayectorias educativas intermitentes o de "baja intensidad" se relacionan estrechamente con el hecho de que las escuelas a las que concurren familias de sectores populares suelen disponer de menores recursos humanos y materiales que aquellas a las que concurren familias de sectores medios y altos, más allá de que sean públicas o no. En 
este sentido, es recurrente que estudiantes de sectores populares mencionen la falta de preparación que tuvieron en sus colegios. Respecto a la universidad, la mayoría coincide en que es más exigente e implica el desarrollo de una mayor autonomía:

\begin{abstract}
Acá tenés que organizarte más vos, ya no llegás si estudiás un rato antes o con lo de las clases. $Y$ si venís de escuelas chotas o si dejaste muchos años de estudiar te cuesta más. Lo mejor es lograr un ritmo de estudio y tratar de mantenerlo, pero a veces se vuelve difícil entre el trabajo y los vaivenes de la vida. (Débora, 22, UBA)

En la secundaria o te están más encima y te malacostumbran, te tratan como nene, o si no te dan muchas horas libres para jugar, te hacen creer que no necesitás aprender nada, que no tiene sentido, o te hacen creer que hablándoles podés convencer a algunos profesores de que te suban la nota. (Micaela, 23, UNLZ)
\end{abstract}

A nivel "académico", también se distinguen problemas respecto a la lectoescritura y a otras áreas de conocimiento que dificultan aún más una transición "exitosa” al ciclo superior, en consonancia con lo que también observa Gallo (2016). Los estudiantes llegan a las universidades con diferentes grados de alfabetización académica, entendida ésta como el conjunto de estrategias y recursos necesarios para participar en la cultura discursiva de cada carrera.
Aprendi a hacer un resumen recién en la facultad. Antes no sabía. $\Upsilon$ me costó mucho porque venía con mala base de la escuela. Pero pude aprovechar talleres, tutorías y clases de apoyo que ofrecía la universidad y eso me sirvió. Además de recursos en internet y estudiar con compañeros de cursada. Me fui armando de recursos y de una base que no tenía para no quedarme afuera. (Julia, 30, UBA)
La secundaria no te involucra en la facultad, no te prepara para un examen o para tomar apuntes en la facultad. Acá en la universidad se lee mucho más, es otra exigencia, otro ritmo, y la secundaria muchas veces no nos prepara para eso. Acá, si no aprovechás bien el tiempo, si no te juntás en grupo antes del examen para repasar, si no estás atento en clase, es muy difícil aprobar. (María, 28, UNLA)

En relación con esto, no se trata sólo de no tener algún familiar universitario, sino que una significativa parte no poseen a nadie cercano, del barrio, la familia o la escuela, que posea "cultura académico-universitaria". En algunos casos, estos referentes académicos suelen ser docentes de la escuela, quienes motivan y brindan herramientas. De este modo, a la cuestión de disponer de menores recursos y mayores exigencias familiares no escolares, en esta población también pesa, en su permanencia y graduación en instituciones de nivel superior, que tienden a carecer de capital social académico.

A las diversas dificultades que enfrenta esta población para integrarse a las instituciones, debe añadirse la menor familiaridad que poseen respecto al lenguaje formal que las instituciones suelen imponer como requisito para su integración satisfactoria. En este sentido, los cursos de ingreso o nivelación que proveen una materia obligatoria de lectoescritura y técnicas de estudio (por ejemplo, la UNLA y la de Hurlingham) junto a ofrecer cursos de ingreso de mayor duración (anuales o cuatrimestrales), generan menores tasas de deserción que los cursos de ingreso intensivos, que duran de uno a dos meses, dependiendo de la institución.

Un error de las instituciones tradicionales es considerar al estudiantado como una tabula rasa sobre la que se imprimen los nuevos conocimientos. Siguiendo esta lógica, la teoría voluntarista meritocrática ("a más estudio, mayor calificación”) invisibiliza las desiguales trayectorias socioeducativas con las que cuenta cada estudiante al momento de ingresar al ciclo superior. Por ejemplo, un joven que fue a un colegio doble jornada bilingüe y además realizó talleres extracurriculares de arte, escritura y cultura, lo que se entiende por una 
experiencia educativa de "alta intensidad", suele contar con una caja de herramientas mayor que otro que realizó una cursada de jornada simple accidentada debido a mudanzas familiares, enfermedades o problemas socioeconómicos.

Además, algunos aspirantes a ingresar a universidades públicas provienen de países sudamericanos con distintas formaciones escolares o han podido terminar el colegio secundario gracias a las denominadas escuelas de reingreso, que existen desde 2004 en la CABA, o al Plan de Finalización de Estudios Primarios y Secundarios (FINES), que funciona de modo gratuito desde 2008 a nivel nacional. Como surge del trabajo de campo, estos estudiantes no tradicionales y con trayectorias discontinuas suelen contar con menor formación académica que quienes provienen de colegios (públicos o privados) en los que una significativa parte de estudiantes pertenece a sectores medios o medio altos.

Terminé la escuela en una escuela de reingreso hace unos años. Al principio me re costó la facultad, pero con paciencia y ayuda de algunas compañeras de las que me hice amiga me fui adaptando. (Romina, 28, UBA)

\begin{abstract}
Me costó adaptarme a la universidad porque había dejado de estudiar hacía varios años porque siempre trabajé muchas horas. Me ayudó la posibilidad de terminar con el Plan Fines y nunca dejar de leer todo lo que podía y tratar de aprovechar el tiempo lo mejor posible. Con la familia y el trabajo a veces no es fácil, pero se puede. Igual vi que un montón de compañeros fueron dejando porque no podían seguir el ritmo, o les costaba un montón cada examen. (Carlos, 30, UNLA)
\end{abstract}

A su vez, he encontrado cierta frustración y desinformación institucional. La mayoría espera que universidad y docentes acompañen y orienten durante el proceso de cursada. Por el contrario, se enfrentan a cierta "frialdad" de la institución, sumado a la falta de información, extensas horas de cursada y, en ocasiones, una oferta horaria poco flexible. Esta frustración, soledad y desinformación institucional que experimentan numerosos estudiantes se acentúa ante las instancias evaluativas tradicionales. En efecto, como también observan Bartolini y Gerlo (2017), la presión que ejercen las clases magistrales y los exámenes finales escritos u orales coadyuvan al desgranamiento.

Como señala la SPU (2016) y Botinelli y Sleiman (2017), la tasa de estudiantes en universidades creció un 20\% entre 2008 y 2015. En el mismo período, la tasa de estudiantes en universidades públicas del Conurbano creció un $20 \%$ en el quintil de mayores ingresos, un $28 \%$ en el cuarto quintil, un $39 \%$ en el tercero, un $95 \%$ en el segundo quintil y un $47 \%$ en el quinto quintil. Estos datos señalan que el crecimiento de la tasa de estudiantes fue significativo en todos los sectores sociales, y en particular en sectores populares.

Pese a esto, la deserción y "sobreedad" continúan siendo una constante, en mayor medida que en otros países de la región. Como explican Botinelli y Sleiman (2017), esto en parte sucede porque en Argentina alrededor de la mitad de estudiantes universitarios trabaja, y un $20 \%$ son jefes de hogar. Por las características más elitistas del resto de los países, este tipo de estudiante que estudia y trabaja suele ser una rara avis. En cambio, la masividad del sistema universitario argentino, que incluye que la mitad de su matrícula sean estudiantes-trabajadores, explica en gran medida las dificultades en torno a la graduación.

\title{
3.3. Casos "exitosos"
}

Se entiende por casos "exitosos" a quienes no abandonan ni son expulsados, sino que logran el objetivo de la graduación, aunque ya de por sí haber llegado a la universidad represente un logro en relación con la generación de sus padres. Pero ¿quiénes son los 
estudiantes de sectores populares que atraviesan con "éxito" la universidad? Según el trabajo de campo, tienden a ser los que desarrollan estrategias para sobrevivir en el sistema académico de sectores medios y altos, apropiándose y "sintiéndose parte" de la universidad. Para lograr el objetivo de graduarse, colocan al estudio como una de sus principales prioridades. Además, tienden a autogestionar y sostener grupos de estudio, que implican contención y apoyo, así como ocupar espacios institucionales y aprovechar los recursos disponibles: becas de estudio y apuntes, bar y comedor estudiantil, salas de estudio, tutorías docentes y de pares, pasantías, clases de apoyo, clases de repaso, herramientas digitales y de la web, bibliotecas, préstamos de libros, foros y actividades extracurriculares.

Me parece que obviamente la universidad tiene cosas por mejorar, pero es la que más
me gustó hasta el momento, ya que pone la inclusión como eje. A diferencia de la UBA,
que me pareció elitista y fría, pese a que también es gratuita. Me gustó de esta
universidad que desde el ingreso hay un montón de actividades gratuitas que propone
la universidad para evitar que los estudiantes abandonen por las dificultades que se
van presentando. (Micaela, 23, UNLZ)

En estos casos se distingue como una constante la perseverancia y la presencia de referentes docentes afectuosos y comprometidos. En línea con lo que sugiere Carli (2012) y Pierella (2016), los docentes más "contenedores" suelen ser quienes dan clases entretenidas, tienen paciencia para repetir explicaciones, escuchan y respetan a los estudiantes, y proponen actividades más horizontales y grupales. Estos permanecen más en la memoria de los estudiantes como ayudas clave para perseverar y "triunfar", en el sentido de lograr finalizar sus carreras.

En parte como consecuencia del mayor involucramiento institucional y aprovechamiento de recursos, y de soportar un mayor tiempo de "inmersión” en los códigos y lenguajes universitarios, los estudiantes de sectores populares también son "exitosos" porque logran desarrollar un interés propio y un placer por la lectoescritura y el conocimiento científicoacadémico. En todo caso, son capaces de desarrollar una metodología de estudio eficiente para aprobar los exámenes necesarios para seguir avanzando y, eventualmente, recibirse.

\begin{abstract}
Aprendí a estudiar en la universidad, porque antes en el colegio eran sólo un par de hojas y listo. O ni eso. Acá tuve que ponerme las pilas y aprender a resumir, resaltar, comprender. Fue medio arrancar de cero. $Y$ fueron claves los tutores para ayudarme en esto. Ahora estoy encaminada y estoy en busca de ser la primera universitaria de la familia. (Julia, 30, UBA)

Ya en el curso de ingreso nos enseñaron metodología de estudio y técnicas de escritura. Después fui a tutorías y con eso me empezó a ir mejor. Al principio reprobé varias materias y pensé en dejar, pero después me encaminé, me ayudó la universidad también. En la UBA y otras universidades sos un número más, acá es distinto. (Mariano, 26, UNDAV)
\end{abstract}

Otro hallazgo de la investigación es que, frente a las dificultades extra-académicas y académicas, las estudiantes mujeres de sectores populares tienen en numerosos casos una mayor determinación y conciencia de sus objetivos y deseos. Muchas han sufrido o sufren distintos tipos de violencia de género y saben o intuyen que necesitan tener más estudios para contar con mayores posibilidades de acceder a un empleo de calidad. Numerosas estudiantes retoman su proyecto universitario después de haber sido madres. Ahora desean ser un buen ejemplo para sus hijos. Estas estudiantes que logran graduarse han resistido y superado toda la "soledad" y presión familiar. Asimismo, la mayoría de la población que utiliza las bibliotecas son estudiantes mujeres. Una de las razones de esto 
es que, como muchas afirman, valoran el espacio de estudio libre de requerimientos hogareños y presiones familiares.

Uso mucho la biblioteca porque no me disperso y puedo consultar libros o información en las computadoras. En mi casa con todas las cosas que hay que hacer y que siempre hay gente cuesta ponerse con el estudio. En la biblioteca nos juntamos con compañeras, llevamos mate y podemos concentrarnos. (Micaela, 23, UNLZ)

La biblioteca es clave porque ahí puedo aislarme por unas horas y concentrarme en lo que tenga que estudiar. Después vuelvo a casa y comienzan los problemas de todos los días y hay que hacer la comida, lavar la ropa, limpiar, etcétera. (Lorena, 26, UNDAV)

A su vez, numerosas entrevistadas sostienen que para ellas la educación universitaria no es sólo una herramienta de movilidad social, sino también de autodefensa y empoderamiento de género. El estudio les permite ser más autónomas y consolidar un proyecto propio, además de pensar fenómenos como la violencia y la desigualdad basada en el género, al tiempo que las ayuda a diseñar estrategias para salir de este tipo de situaciones o, al menos, responder mejor a ellas (Linne y Villamil, 2016).

\section{Discusión y conclusiones}

Este trabajo se ha propuesto ampliar y complejizar la mirada en torno a la población de estudiantes universitarios de sectores populares. Entre sus limitaciones, dada la escasez de la muestra y la dificultad para encontrar datos estadísticos sobre el universo analizado, los resultados no son extrapolables al conjunto de estudiantes en cuestión. Queda pendiente para futuros estudios poder realizar una muestra más amplia, que permita dar mayor extensión a los resultados obtenidos. Entre los aportes realizados, este artículo profundiza en la discusión del estado de la cuestión, e identifica algunas de las principales razones por las que estos grupos de estudiantes tienden a tener mayores dificultades para permanecer como estudiantes de nivel superior y, en mayor medida, para convertirse en graduados universitarios. En este sentido, ha quedado evidenciado que una significativa parte de los estudiantes de sectores populares consultados perciben mayor ajenidad u hostilidad frente al sistema y tienden a realizar trayectorias intermitentes. A su vez, otro aporte del estudio es distinguir razones académicas y extra-académicas entre las principales causas que contribuyen a la deserción de estudiantes de sectores populares.

En términos bourdieanos, existe un capital cultural que, sobre todo en las clases populares, los estudiantes no detentan. En relación con esto, numerosos estudiantes se autoculpabilizan, considerando no sentirse motivados o no ser aptos para el estudio universitario ("no tenía ganas", "no me da la cabeza", "no era lo mío", "nunca tuve madera para el estudio"). Las universidades suelen reprobarlos y expulsarlos progresivamente con el argumento meritocrático, a través de la exigencia académica y la reiterada reprobación de exámenes sobre contenidos no vistos o no trabajados suficientemente en colegios, cursos de ingreso y materias iniciales.

Pese a las múltiples dificultades que enfrenta esta población para ingresar, permanecer y graduarse en instituciones universitarias, las mujeres suelen poseer una mayor determinación en su proyecto académico, que asocian a una mayor autonomía y empoderamiento de género. El trabajo de campo evidencia que los servicios de tutorías docentes y pares, los espacios de consultas, las bibliotecas y los lugares de estudio son en mayor medida utilizados por mujeres. Esta sería una de las variables "independientes" que explican en parte a los casos exitosos. Sin embargo, la tasa de graduación es mayor en 
varones que en mujeres, lo que se explica en parte porque ellas suelen ser quienes más cargas familiares y domésticas portan.

Los estudiantes de sectores populares que atraviesan con éxito la universidad suelen desarrollar estrategias más eficientes para sobrevivir en el sistema. Para esto, se animan a pedir ayuda y enfrentan la inseguridad o el pudor, porque de algún modo saben que la institución también les pertenece. Las principales actividades que realizan para apropiarse y "sentirse parte" de la universidad son autogestionar y sostener grupos de estudio, ocupar espacios y aprovechar estratégicamente los recursos disponibles de sus instituciones.

Al mismo tiempo, observé que los centros de estudiantes representan espacios de activa afiliación institucional inter pares. Quienes participan activamente del centro de estudiantes -y quienes son beneficiarios de becas de ayuda económica- en numerosos casos trabajan ad honorem como agentes de afiliación institucional para la universidad, dando clases de apoyo, tutorías o cooperando en actividades de extensión, en consonancia con los estudios de Blanco (2014).

Esta serie de universidades públicas del Conurbano, que suelen ser denominadas como más "inclusivas", han generado distintos mecanismos institucionales en pos de disminuir la deserción y aumentar la afiliación institucional. En efecto, el uso de diversos recursos y estrategias son mencionados por los estudiantes de sectores populares que han logrado graduarse o se encuentran en un estadio avanzado de sus carreras.

No obstante, estas instituciones suelen proveer más herramientas para la inclusión y el acceso que para su graduación. Ante la menor presencia de mecanismos institucionales para colaborar en la graduación de quienes poseen menor cultura académica, se genera cierto cuello de botella en el que numerosos estudiantes de sectores populares llegan a las instancias finales de una carrera y les cuesta recibirse por dificultad para encarar las tesinas o trabajos finales integradores, o para realizar la pasantía necesaria. Esto no implica que miles de estudiantes de sectores populares, ayudados por recursos institucionales, sí logren graduarse.

En algunos casos, las universidades públicas del Conurbano son acusadas, tanto por egresados de universidades privadas como de universidades públicas tradicionales, de ser centros culturales, de poseer un menor rigor formativo y otorgar títulos con menores exigencias académicas que las casas de estudio tradicionales. En consonancia con los desarrollos de Mónaco y Herrera (2015), estas acusaciones descalifican al mismo tiempo a la mayoría de estudiantes que concurren a estas instituciones, que suelen ser de sectores populares.

Las mencionadas críticas se fundan en parte en un enfoque liberal, de élites y economicista. Santos Sharpe y Carli (2016) afirman que los estudios de corte economicista ven a la educación superior como una mera inversión económica y un gasto, caracterizan al sujetoestudiante como un individuo autónomo y completamente responsable, culpabilizándolo de sus dificultades académicas, y diagnostican el abandono universitario como un costo social.

Estos enfoques reduccionistas, que sólo evalúan como inversión positiva el número de graduados, olvidan que las universidades son, además de dispositivos de graduación de profesionales, espacios de contención y formación juvenil y adulta, así como centros de extensión y recreación, instituciones de investigación científica, ámbitos de producción, 
discusión y divulgación cultural, instancias de intercambio interdisciplinar, artístico y social.

En línea con trabajos como el de Sito y Kleiman (2017), se distingue una singular representación de la universidad en sectores populares. A diferencia del imaginario que suelen compartir estudiantes hijos de graduados universitarios, en sectores populares la universidad les resulta expulsiva y de élites, ajena a sus mundos de vida cotidianos. Para contrarrestar estos imaginarios juveniles, en el Conurbano se crearon universidades públicas y gratuitas con el fin de democratizar el acceso al conocimiento, con la certeza de que esto contribuirá a crear una sociedad más igualitaria y democrática.

El problema es que, pese a los avances institucionales y a la ampliación de las tasas de acceso y permanencia en el sistema educativo, las tasas de graduación universitaria de los sectores populares continúan siendo relativamente bajas. Si el Estado y las instituciones vinculadas a la educación superior poseen el objetivo de contribuir a una sociedad más inclusiva, es menester profundizar en el análisis de los procesos que contribuyen o dificultan la experiencia académica de estas poblaciones, históricamente relegadas.

\section{Referencias}

Arias, M., Mihal, I., Lastra, K. y Gorostiaga, J. (2014). El problema de la equidad en las universidades del conurbano bonaerense en Argentina. Un análisis de políticas institucionales para favorecer la retención. Revista Mexicana de Investigación Educativa, 2O(64), 47-69.

Bartolini, A. y Gerlo, G. (2017). Reflexiones sobre experiencias de abandono de escuela secundaria y doctorado en Argentina. Modos de cierre y condicionantes individuales e institucionales. Universitas Humanística, 83, 85-108.

Botinelli, L. y Sleiman, C. (2017). Un mapa en expansión. Le Monde Diplomatique, 2, 4-6.

Bourdieu, P. y Passeron, J. C. (2003). Los herederos. Los estudiantes y la cultura. Buenos Aires: Siglo XXI.

Blanco, R. (2014). Universidad intima y sexualidades públicas. Buenos Aires: Miño y Dávila.

Bracchi, C. (2016). Descifrando el oficio de ser estudiantes universitarios: entre la desigualdad, la fragmentación y las trayectorias educativas diversificadas. Trayectorias Universitarias, 2(3), $3-14$.

Busso, M. y Pérez, P. (2015). Combinar trabajo y estudios superiores ¿Un privilegio de jóvenes de sectores de altos ingresos? Población y Sociedad, 22(1), 5-29.

Carabaña, J. (2013). Crecimiento del bachillerato e igualdad desde los años ochenta. RASE: Revista de la Asociación de Sociología de la Educación, 6(1), 6-31.

Carli, S. (2012). El estudiante universitario. Buenos Aires: Siglo XXI.

Carlino, P. (2005). Escribir, leer y aprender en la universidad. Una introducción a la alfabetización académica. Buenos Aires: Fondo de Cultura Económica.

Chaves, M., Fuentes, S. y Vecino, L. (2016). Experiencias juveniles de la desigualdad. Fronteras y merecimientos en sectores populares, medios y altos. Buenos Aires: Grupo Editor Universitario.

Chiroleu, A. (2016). Políticas públicas de inclusión y democratización universitaria. En I. Aranciaga (Ed.), El desafío de construir sociedades inclusivas (pp. 10-39). Río Gallegos: Universidad Nacional de la Patagonia Austral. 
Coulon, A. (2005). Le métier d'étudiant. L'entrée dans la vie universitaire. París: Anthropos.

Daza, L. (2013). Capital social y aprendizaje en la universidad. Barcelona: Universitat de Barcelona.

Elias, M. (2008). Los abandonos universitarios: Retos ante el espacio europeo de educación superior. Estudios sobre Educación, 15, 101-121.

Ezcurra, A. M. (2011). Igualdad en educación Superior. Un desafio mundial. Buenos Aires: UNGS.

García de Fanelli, A. (2014). Inclusión social en la educación superior argentina: Indicadores y políticas en torno al acceso y a la graduación. Páginas de Educación, 7(2), 124-151.

García de Fanelli, A. y Adrogué, C. (2015). Abandono de los estudios universitarios: Dimensión, factores asociados y desafíos para la política pública. Fuentes, 16, 85-106. https://doi.org/10.12795/revistafuentes.2015.i16.04

Gallo, L. (2016). Aprender el oficio de estudiante universitario. Trayectorias Universitarias, 2(3), 8188.

Gorostiaga, J., Lastra, K. y Muiños de Britos, S. (2017). Políticas institucionales para favorecer el acceso y la permanencia en universidades argentinas: Un análisis de cuatro instituciones del conurbano bonaerense. Páginas de Educación, 10(1), 151-173. https://doi.org/10.22235/pe.v10i1.1363

Glaser, B. y Strauss, A. (1967). The discovery of grounded theory: Strategies for qualitative research. Nueva York, NY: Aldine Publishing Company.

Gluz, N. (2011). Admisión a la universidad y selectividad social. Cuando la democratización es más que un problema de “ingresos”. Buenos Aires: UNGS.

Grandoli, M. (2011). Aproximación al estudio de las dificultades académicas como condicionantes de desempeño y permanencia en los estudiantes ingresantes al CAU. En N. Gluz (Ed.), Admisión a la universidad y selectividad social. Cuando la democratización es más que un problema de “ingresos" (pp. 203-230). Buenos Aires: UNGS.

Habley, W. y McClanahan, R. (2004). What works in student retention? All survey colleges. Iowa, US: American College Testing Program. https://doi.org/10.1037/e420532008-001

INDEC. (2012). Encuesta nacional sobre el acceso y uso de las TIC. Buenos Aires: INDEC.

Kessler, G. (2002). La experiencia educativa fragmentada. Estudiantes y docentes en la escuela media en Buenos Aires. Buenos Aires: IIPE.

Laing, C. y Robinson, A. (2003). The withdrawal of non-traditional students: Developing an explanatory model. Journal of Further and Higher Education, 27(2), 175-185. https://doi.org/10.1080/0309877032000065190

Linne, J. y Villamil, L. (2016). El sentido de estudiar. Revista Turba, 6, 56-57.

Manuale, M. (2013). El oficio de estudiante universitario y el problema del ingreso: Miradas y aportes desde el gabinete pedagógico de la facultad de bioquímica y ciencias biológicas. Revista Aula Universitaria, 15, 43-57.

Margulis, M. y Urresti, M. (1996). La juventud es más que una palabra. Buenos Aires: Biblos.

Mayer, L. y Cerezo, L. (2016). Tutorías y estipendio mensual: contribuciones a la trayectoria universitaria de jóvenes en situación de vulnerabilidad. Revista Latinoamericana de Ciencias Sociales, Niñez y Juventud, 14(2), 1421-1433.

Milesi, C. (2010). Do all roads lead to Rome? Effect of educational trajectories on educational transitions. Research in Social Stratification and Mobility, 28(1), 23-44. https://doi.org/10.1016/j.rssm.2009.12.002 
Mónaco, J. y Herrera, D. (2015). ¿Quiénes son los nuevos estudiantes universitarios? Le Monde Diplomatique, 30, 5-9.

Nobile, M. (2016). La escuela secundaria obligatoria en Argentina: Desafíos pendientes para la integración de todos los jóvenes. Última década, 44, 109-131.

Núñez, P. y Litichever, L. (2016). Ser joven en la escuela: temporalidades y sentidos de la experiencia escolar en la Argentina. Psicoperspectivas, 16(2), 91-102.

Olave, G., Rojas, I. y Cisneros, M. (2013). Deserción universitaria y alfabetización académica. Educación y Educadores, 16(3), 455-471.

Otero, A. y Corica, A. (2017). Jóvenes y educación superior en Argentina. Evolución y tendencias. Revista Interamericana de Educación de Adultos, 39(1), 11-25.

Pierella, P. (2016). Los exámenes en el primer año de la universidad. ¿Instancia de formación o mecanismo de selectividad social? Trayectorias Universitarias, 2(2), 11-19.

Rojas, M. (2009). El abandono de los estudios: deserción y decepción de la juventud. Hologramática, $10(4), 75-94$.

Sánchez-Gelabert, A. y Andreu, M. (2017). Los estudiantes universitarios no tradicionales y el abandono de los estudios. Estudios Sobre Educación, 32, 27-48. https://doi.org/10.15581/004.32.27-48

Santos Sharpe, A. y Carli, S. (2016). Estudios globales y locales sobre el abandono de los estudios universitarios. Teorías, perspectivas y nuevos abordajes. Revista Argentina de Educación Superior, 8(13), 6- 31 .

Saraví, G. (2015). Juventudes fragmentadas. Socialización, clase y cultura en la construcción de la desigualdad. Ciudad de México: Flacso.

SPU. (2016). Estadísticas universitarias argentinas. Buenos Aires: Departamento de Información Estadística.

Sito, L. y Kleiman, A. (2017). Eso no es lo mío: Un análisis de conflictos en la apropiación de prácticas de literacidad académica. Universitas Humanística, 83, 159-185.

Tinto, V. (2005). Epilogue. Moving from theory to action. En A. Seidman (Ed.), College student retention (pp. 317-334). Westport: Praeger Publishers.

Tiramonti, G. (Comp.). (2004). La trama de la desigualdad educativa. Mutaciones recientes en la escuela media. Buenos Aires: Manantial.

Urresti, M., Linne, J. y Basile, D, (2015). Conexión total. Los jóvenes y la experiencia social en la era de la comunicación digital. Buenos Aires: Grupo Editor Universitario.

Wallach, W. y Vidondo, M. (2017, marzo). Dispositivos de acompañamiento en el ingreso: la experiencia en la Universidad Nacional de Hurlingham. Comunicación presentada en el VII Encuentro Nacional y VI Encuentro Latinoamericano sobre Ingreso Universitario. Universidad Nacional de Cuyo.

Zandomeni, N., Canale, A. Pacifico, A. y Pagura, F. (2016). El abandono en las etapas iniciales de los estudios superiores. Ciencia, Docencia y Tecnología, 27(52), 127-152. 


\section{Breve CV del autor}

\section{Joaquín Linne}

Sociólogo, Magíster en Comunicación y Cultura y Doctor en Ciencias Sociales. Investigador del Consejo Nacional de Investigaciones Científicas y Técnicas (CONICET) con sede en el Instituto Gino Germani de la Universidad de Buenos Aires (UBA). Docente-investigador de la Universidad Nacional de Lanús (UNLa) y de la UBA. Desde 2015 da clases en los cursos de ingreso de ambas instituciones. Ha publicado un libro en coautoría y diversos artículos sobre jóvenes de sectores populares, educación y tecnologías de la información y comunicación. Participa de grupos de investigación en estas instituciones y en Flacso, donde continúa indagando sobre estas problemáticas. ORCID ID: 0000-0002-4297-3914. Email: joaquinlinne@gmail.com 